\title{
Problems on Yield Criterion and Dilatancy of Limit Analysis Finite Elements to Bearing Capacity of Geomaterials
}

\author{
Wei-Xue Kong ${ }^{1,2, *}$, Ying-Ren Zheng $^{3}$ and Lu-Hui Yan ${ }^{2}$ \\ ${ }^{I}$ Engineering Research Center of Catastrophic Prophylaxis and Treatment of Road \& Traffic Safety (Changsha \\ University of Science \& Technology), Ministry of Education, Changsha 410004, China \\ ${ }^{2}$ School of Basic Education for Commanding Officers, National University of Defense Technology, Changsha 410072, \\ China \\ ${ }^{3}$ Department of Architecture and Civil Engineering, Logistical Engineering University, Chongqing 401311, China
}

\begin{abstract}
The theoretical basis of classic geotechnical engineering stability problems is limit analyis thereom. Incremental loading finite elements and strength reduction finite elements were put forward by Zienkiewicz in 1975 and the methods are called by the authors Limit Analysis Finite Elements (abbreviation LAFE for short). It has been successfully applied to slope engineering, and used to bearing capacity problems foundations. The LAFE method is still in initial stage, with problems in engineering practice. Key problems on yield criterion and dilatancy angle were also discussed in detail. The paper proved again that same ultimate bearing capacity and slip line are obtained in slip line field theory under associated and nonassociated flow rule, with the only difference of velocity vector direction. Meanwhile, the dilatancy angle should be $\varphi / 2$ when nonassociated flow rule is employed under plane strain, and corresponding volumetric strain is zero. Thus the correctness of the theoretical solution in literature [19] is proved, and LAFE method is also proved a very promising approach in solving bearing capacity problems of foundations. Rigorous theoretical basis is available for finite elements incremental loading to solve the bearing capacity problems of foundations, and the approach is simple to use. In the numerical simulation process, not only the ultimate bearing capacity and load-displacement curve are obtained, but also the failure mechanism proved same as the one by traditional limit analysis approach is achieved. Only the yield criterion matched with practical engineering problems can generate a precise result. Under plane strain the results by MohrCoulomb inscribed circle yield criterion (DP3) for associated flow rule, and Mohr-Coulomb match yield criterion (DP5) for nonassociated flow rule are close to the accurate theoretical solution by Prandtl. The achievements can be applied in practical geotechnical engineering purposes.
\end{abstract}

Keywords: Limit analysis finite elements, Geomaterials, Dilatancy angle, Yield criterion, Slip line field, Ultimate Bearing capacity of foundation, Collapse mechanism.

\section{INTRODUCTION}

Classic geotechnical engineering stability problems include slope stability, bearing capacity of foundations and earth pressure problems, and limit analyis thereom [1,2] is their theoretical basis. Limit analysis theory starts from Coulomb law of 1773. In 1920s, Fellenius etc founded limit equilibrium method, and then slip line field theory was founded in succession by Sokolovskii in 1940s. In 1950s, upper and lower bound limit analysis were then founded [3]. After 100 years' development, limit analysis method has gradually become mature. It demonstrated good practica-ility in engineering practice, and solved some problems in engineering design, especially strength and stability problems.

*Address correspondence to this author at the Engineering Research Center of Catastrophic Prophylaxis and Treatment of Road \& Traffic Safety, Changsha University of Science \& Technology, Ministry of Education, Changsha 410004, China; Tel: (86) 731-85258786;

E-mail: kong_weixue@sina.com
Due to difficulties in solving traditional limit analysis equations, discrete methods, such as slip line field finite difference, upper and lower bound finite elements etc, were applied to limit analysis theorem. Meanwhile, incremental loading finite elements and strength reduction finite elements were put forward by Zienkiewicz in 1975 [2,4], which were called by the authors Limit Analysis Finite Elements $[2,5,6]$ (abbreviation LAFE for short) or limit analysis numerical simulation. LAFE methods see a bright future due to its high accuracy, conveniency and great applicability and practicability, even though now currently mainly used in slope stability analysis.

Two alternatives are available for geomaterials to reach limit equilibrium state through finite elements method. One is to apply gradually increasing load, called incremental loading finite elements, and another is to reduce gradually strength parameters, called strength reduction finite elements. Incremental loading finite elements should be applied to the solution of ultimate load of foundations. The applica- 
tion of strength reduction finite elements will be discussed in another paper.

In finite elements analysis under limit equilibrium state, slip line, and velocity vector direction, and ultimate load can be obtained automatically. Thus further work can be done to prove the correctness of the theoretical solutions under nonassociated and associated flow rule in slip line field theory.

The essence of LAFE is that geomaterials reach failure state through reducing strength indexes or increasing applied load. The failure surface is then automatically obtained, and the corresponding safety factor can also be obtained. Different from traditional methods, LAFE can offer failure surface automatically, without assuming failure surface. Academican Zheng Y R of Chinese Academy of Engineering etc put forward approaches to determine the failure surfaces in finite elements analysis. Unfortunately, the function is unavailable in current finite elements software, the failure surface needs solving by hand. So it can be seen that the essence of LAFE is still a limit analysis method, just with a solution through numerical simulation tool. Ultimate load and stability safe factor can be directly acquired by computation without assuming sliding surface, or seeking sliding force and skid resistance on failure surface. The position and shape of the failure surface can also be determined in computation. So the function and the extent to which applied is widely expanded.

It should be mentioned that LAFE method is still in initial stage, with problems in engineering practice. For example, effective criterion for failure of geomaterials in LAFE computation should be found, or the operator doesn't know that even though the computer has automatically found failure surface and shown failure state, and geomaterials has failed. Or for some reason, the computer cannot solve influently, which caused that geomaterials cannot reach failure. The possible reason is that irrational mesh causes computation unconvergence, especially mesh distortion after strength reduction makes solutions unavailable. Similar problems should be tackled step by step in numerical simulation practice. Rational and effective criterion for LAFE should be sought, in spite of its conveniency in solving. There is no rigorous proof for current criterion, even though they spring from mechanical analysis, which has led to its confinement in application. Further work should be implemented for complicated cases.

The LAFE method was applied to foundation bearing capacity problems. Key problems on yield criterion and dilatancy angle were also discussed in detail in this paper.

\section{SELECT OF YIELD CRITERION}

Ideal elastic plastic method is adopted for constitutive model of geomaterials in strength reduction finite elements. Different yield criteria can be adopted and corresponding safety factors are in close form with the yield criterion. Different safety factors can be obtained under different yield criteria.

Mohr-Coulomb(MC) criterion is mostly adopted, and demonstrates some shortcomings. For example, it doesn't consider intermediate principal stress. It is a discontinuous function in 3-Dimensional stress space, and its yield surface is an angle cone surface of irregular hexagon (Fig. 1). Moreover, the yield curve on deviation plane ( $\pi$ plane) is an irregular hexagon (Fig. 2) with fastigium and wracking, which bring great difficulty to numerical simulation in plastic theory computation. In the computation normal vector of yield surface is needed.

The yield surface of Drucker-Prager (DP) yield criterion in 3-Dimensional principal stress space is smooth cone plane (Fig. 3), and its yield curve on $\pi$ plane is a circle, without fastigium problem, which makes a high efficiency in numerical simulation. So Drucker-Prager yield criterion is widely adopted in large finite elements softwares, such as ANSYS, MARC, NASTRAN etc. Its foumula is:

$$
F(\sigma)=\alpha I_{1}+\sqrt{J_{2}}=\kappa
$$

where

$$
\begin{aligned}
& I_{1}=\sigma_{1}+\sigma_{2}+\sigma_{3} \\
& J_{2}=\frac{1}{6}\left[\left(\sigma_{1}-\sigma_{2}\right)^{2}+\left(\sigma_{1}-\sigma_{3}\right)^{2}+\left(\sigma_{2}-\sigma_{3}\right)^{2}\right]
\end{aligned}
$$

Here $I_{1}$ and $J_{2}$ are first invariant of stress tensor and second invariant of stress deviator respectively. $\alpha$ and $\kappa$ are constants concerning strength indexes $c$ (cohesion) and $\varphi$ (internal friction angle). Different $\alpha$ and $\kappa$ present different circles on $\pi$ plane (Fig. 2). DP series criteria can be realized by transfering $\alpha$ and $\kappa$ (Table 1) [7-9].

The criterion was first advanced by Drucker and Prager in 1952, so it is called Drucker-Prager criterion, or generalized Von Mises yield criterion. The criterion degraded to Von Mises yield criterion when $c=0$.

The adopted yield criterion in the software of ANSYS is MC irregular hexagon circumscribed circle yield criterion (DP1 in Table 1). Rearches showed that the result obtained through the criterion creates a large error compared with that by traditional MC yield criterion, and the results in practical engineering design employing DP1 criterion is insecure.

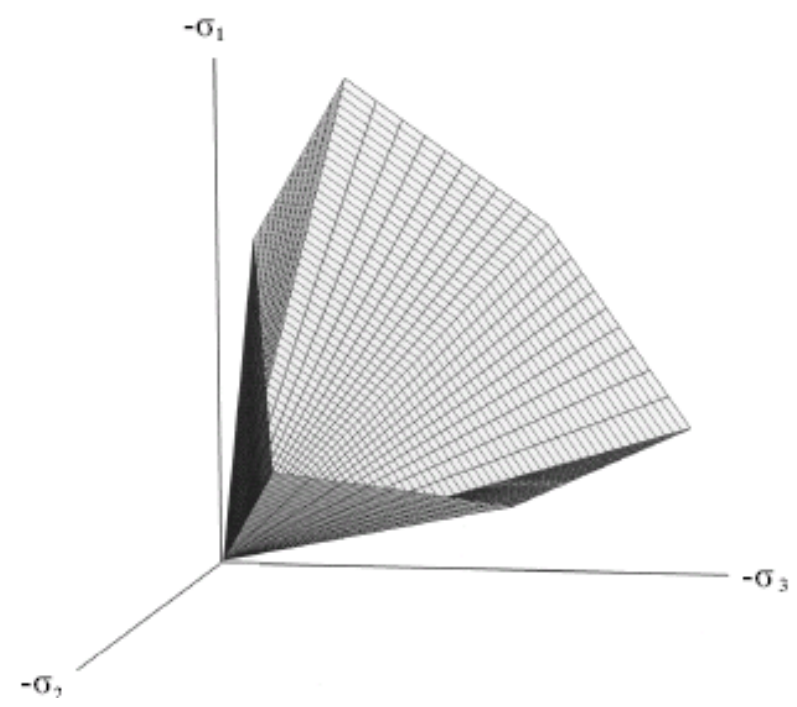

Fig. (1). Mohr-Coulomb yield plane in 3-dimensional space. 


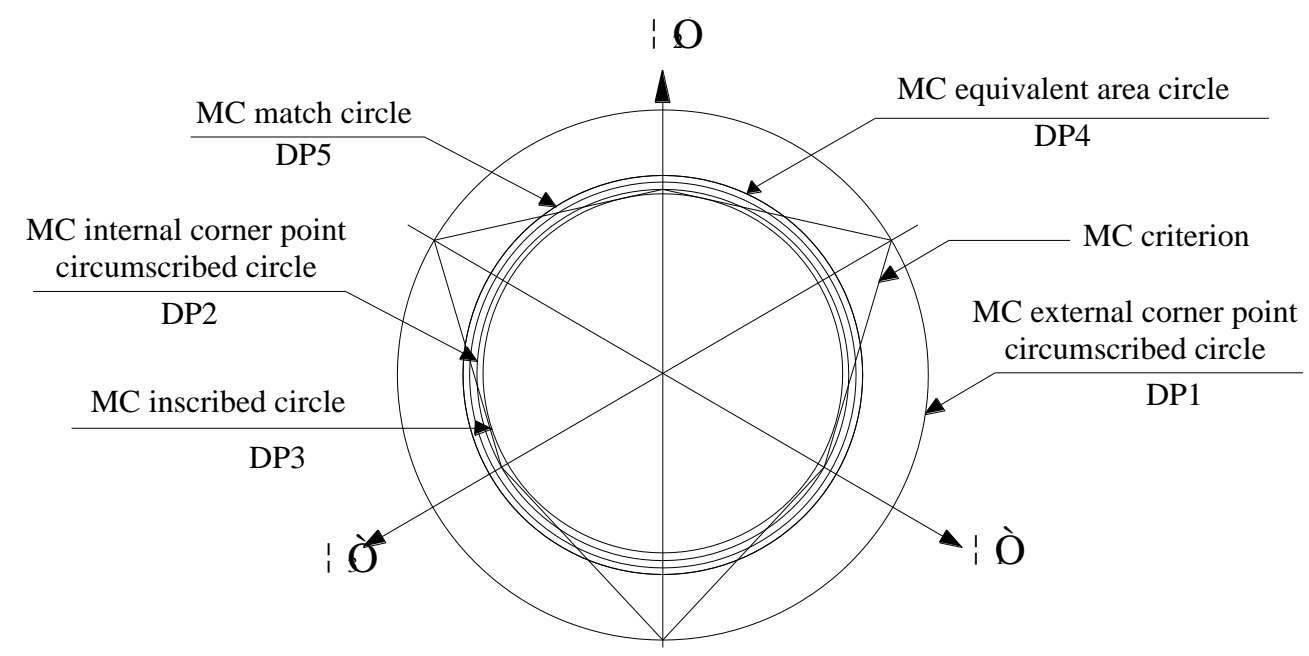

Fig. (2). Deviatoric sections of Mohr-Coulomb (MC) and Drucker-Prager (DP) criterion series.

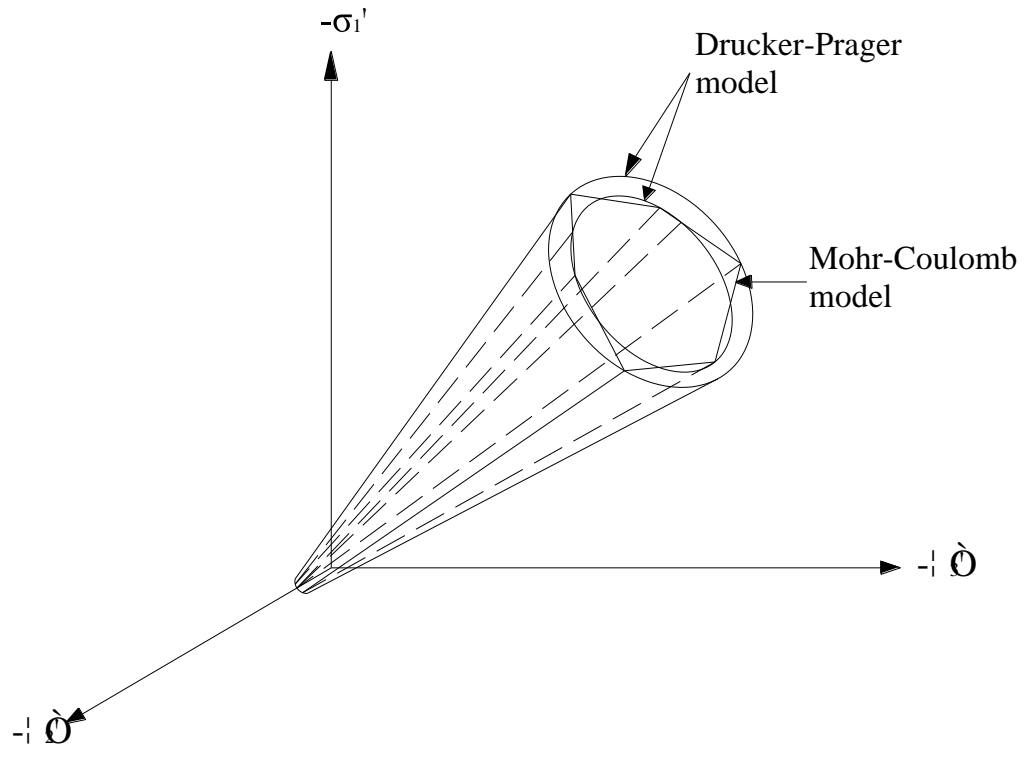

Fig. (3). Drucker-Prager yield surface in 3-dimensional space.

Table 1. Conversions Among DP Criteria

\begin{tabular}{|c|c|c|c|}
\hline No & Criterion Type & $\alpha$ & $\kappa$ \\
\hline \hline DP1 & MC external corner point circumscribed circle & $2 \sin \phi /[\sqrt{3}(3-\sin \phi)]$ & $6 c \cos \phi /[\sqrt{3}(3-\sin \phi)]$ \\
\hline DP2 & MC internal corner point circumscribed circle & $2 \sin \phi /[\sqrt{3}(3+\sin \phi)]$ & $6 c \cos \phi /[\sqrt{3}(3+\sin \phi)]$ \\
\hline DP3 & MC inscribed circle & $\sin \phi /\left[\sqrt{3} \sqrt{3+\sin ^{2} \phi}\right]$ & $3 c \cos \phi /\left[\sqrt{3} \sqrt{3+\sin ^{2} \phi}\right]$ \\
\hline DP4 & MC equivalent area circle & $2 \sqrt{3} \sin \phi / \sqrt{2 \sqrt{3} \pi\left(9-\sin ^{2} \phi\right)}$ & $6 \sqrt{3} c \cos \phi / \sqrt{2 \sqrt{3}} \pi\left(9-\sin ^{2} \phi\right)$ \\
\hline DP5 & MC match circle & $\sin \phi / 3$ & $c \cos \phi$ \\
\hline
\end{tabular}

Zhao S Y ([10], 2004) thinks that MC equivalent area circle criterion(DP4), advanced by Xu G C and Zheng Y R ([11],1990), is of high accuracy in slope stability analysis using finite elements instead of traditional MC yield criterion. Results show that the average error of safety factors obtained by FEM with Mohr-Coulomb equivalent area circle DP yield cirterion and by Spencer method is about $5 \%$.
The DP4 criterion apply to 2-Dimensional or 3Dimensional computation. Under plane strain, MC match yield criterion(DP5), matched with traditional MohrCoulomb criterion, may be used. MC criterion is as follows in 2-Dimensional stress space (Fig. 4):

$$
\tau=c+\sigma_{n} \tan \phi
$$




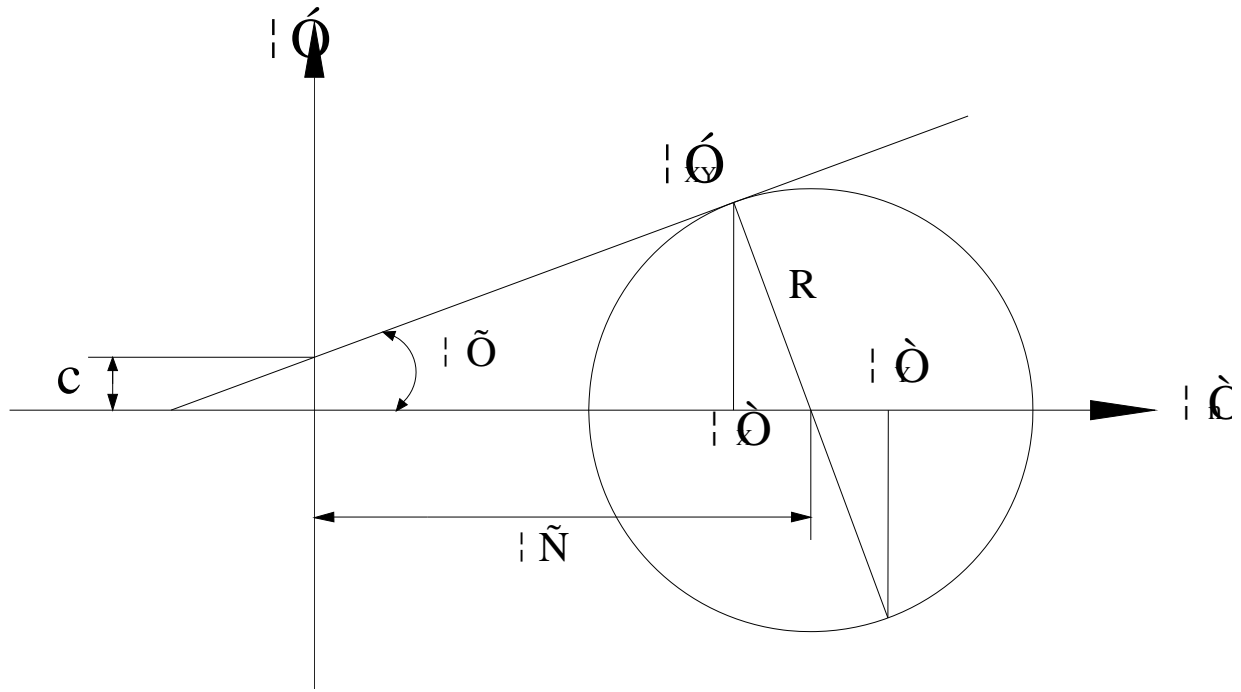

Fig. (4). Mohr-Coulomb yield criterion.

$$
R=c \times \cos \phi+\rho \sin \phi=\sqrt{\left(\sigma_{X}-\sigma_{Y}\right)^{2} / 4+\tau_{X Y}^{2}}
$$

Here $\rho=\left(\sigma_{X}+\sigma_{Y}\right) / 2$.

DP criterion in 3-Dimesional stress space is as shown in (Eq. 1).

Yield function equals to plastic potential function under associated flow rule, that is $F=Q$. Here, flow vector $r$ may be as follows:

$r_{i j}=\frac{\partial F}{\partial \sigma}=a_{\phi} \delta_{i j}+\frac{1}{2 \sqrt{J_{2}}} s_{i j}, \quad$ in which $\delta_{i j}=\left\{\begin{array}{ll}1 & i=j \\ 0 & i \neq j\end{array}\right\}$

As for nonassociated flow rule, $F \neq Q$. Suppose $Q$ has the same form as $F$, with $a_{\psi}$ instead of $a_{\phi}$.

$r_{i j}=\frac{\partial Q}{\partial \sigma}=a_{\psi} \delta_{i j}+\frac{1}{2 \sqrt{J_{2}}} s_{i j}$

For ideal elastic plastic material after plastic yield state, elastic strain increment $d \varepsilon^{e}=0$. Here, suppose elastic strain remains unchanged. Total strain increment equals to total plastic strain increment, that is $d \varepsilon=d \varepsilon^{p}$. Under plane strain, $d \varepsilon_{Z}^{p}=d \varepsilon_{X Z}^{p}=d \varepsilon_{Y Z}^{p}=0$. According to flow equation:

$d \varepsilon_{i j}^{p}=d \lambda \frac{\partial Q}{\partial \sigma}=d \lambda\left(a_{\psi} \delta_{i j}+\frac{1}{2 \sqrt{J_{2}}} s_{i j}\right)$, we have

$d \varepsilon_{Z}^{p}=d \lambda \frac{\partial Q}{\partial \sigma}=d \lambda\left(a_{\psi}+\frac{1}{2 \sqrt{J_{2}}} S_{Z}\right)=0$

$d \varepsilon_{X Z}^{p}=d \lambda \frac{\partial Q}{\partial \sigma}=d \lambda \frac{1}{2 \sqrt{J_{2}}} S_{X Z}=0$

$d \varepsilon_{Y Z}^{p}=d \lambda \frac{\partial Q}{\partial \sigma}=d \lambda \frac{1}{2 \sqrt{J_{2}}} S_{Y Z}=0$
Then $\quad S_{Z}=-2 a_{\psi} \sqrt{J_{2}}=S_{3}=\sigma_{3}-\frac{I_{1}}{3}$,

$S_{X Z}=\tau_{X Z}=S_{Y Z}=\tau_{Y Z}=0$

As $I_{1}=\sigma_{1}+\sigma_{2}+\sigma_{3}=\sigma_{1}+\sigma_{2}+S_{3}+\frac{I_{1}}{3}$ or

$I_{1}=\frac{3}{2}\left(\sigma_{1}+\sigma_{2}\right)+-3 a_{\psi} \sqrt{J_{2}}$

$\sigma_{Z}=S_{3}+\frac{I_{1}}{3}=-2 a_{\psi} \sqrt{J_{2}}+\frac{1}{2}\left(\sigma_{1}+\sigma_{2}\right)-a_{\psi} \sqrt{J_{2}}=\frac{1}{2}\left(\sigma_{1}+\sigma_{2}\right)-3 a_{\psi} \sqrt{J_{2}}$

Because $\sigma_{1}+\sigma_{2}=\sigma_{X}+\sigma_{Y}$, we have

$\sigma_{X}-\sigma_{Z}=\sigma_{X}-\frac{1}{2}\left(\sigma_{1}+\sigma_{2}\right)+3 a_{\psi} \sqrt{J_{2}}=\frac{1}{2}\left(\sigma_{X}-\sigma_{Y}\right)+3 a_{\psi} \sqrt{J_{2}}$

Similarly

$\sigma_{Y}-\sigma_{Z}=\frac{1}{2}\left(\sigma_{Y}-\sigma_{X}\right)+3 a_{\psi} \sqrt{J_{2}}$

available,

$$
\begin{aligned}
& \text { So } \\
& J_{2}=\frac{1}{6}\left[\left(\sigma_{X}-\sigma_{Y}\right)^{2}+\left(\sigma_{X}-\sigma_{Z}\right)^{2}+\left(\sigma_{Y}-\sigma_{Z}\right)^{2}+6 \tau_{X Y}^{2}+6 \tau_{Y Z}^{2}+6 \tau_{X Z}^{2}\right] \\
& J_{2}=\frac{1}{6}\left[\frac{3}{2}\left(\sigma_{X}-\sigma_{Y}\right)^{2}+18 a_{\psi}^{2} J_{2}+6 \tau_{X Y}^{2}\right]
\end{aligned}
$$

then

$$
J_{2}=\frac{\frac{1}{4}\left(\sigma_{X}-\sigma_{Y}\right)^{2}+\tau_{X Y}^{2}}{1-3 a_{\psi}^{2}}=\frac{R^{2}}{1-3 a_{\psi}^{2}}
$$

Substitution of $I_{1}$ and $J_{2}$ to (Eq. 1),

$$
\frac{3}{2} a_{\phi}\left(\sigma_{X}+\sigma_{Y}\right)+\frac{R\left(1-3 a_{\phi} a_{\psi}\right)}{\sqrt{1-3 a_{\psi}^{2}}}-k=0
$$




$$
R=\frac{\sqrt{1-3 a_{\psi}^{2}}}{1-3 a_{\phi} a_{\psi}}\left[-\frac{3}{2} a_{\phi}\left(\sigma_{X}+\sigma_{Y}\right)+k\right]
$$

Considering Eq. 2 and Eq. 12, we have

$$
\begin{aligned}
& \sin \phi=3 a_{\phi} \frac{\sqrt{1-3 a_{\psi}^{2}}}{1-3 a_{\phi} a_{\psi}} \\
& c \cos \phi=k \frac{\sqrt{1-3 a_{\psi}^{2}}}{1-3 a_{\phi} a_{\psi}}
\end{aligned}
$$

After further transform, we have

$$
\begin{aligned}
& a_{\phi}=\frac{\sin \phi}{3}\left(a_{\psi} \sin \varphi+\frac{1}{\sqrt{1-3 a_{\psi}^{2}}}\right) \\
& k=c \cos \phi\left(a_{\psi} \sin \phi+\frac{1}{\sqrt{1-3 a_{\psi}^{2}}}\right)
\end{aligned}
$$

When nonassociated flow rule adopted, $a_{\psi}=0$, and we have

$$
a_{\phi}=\frac{\sin \phi}{3}
$$

$k=c \cos \phi$

The dilatancy angle is unavailable in formula derivation and is often given the value 0 . The problem concerning the dilatancy angle will be discussed in detail in the following section.

When associated flow rule adopted, $a_{\psi}=a_{\varphi}$. We have

$$
\begin{aligned}
& a_{\phi}=\frac{\tan \phi}{\sqrt{9+12 \tan ^{2} \phi}}=\frac{\sin \phi}{\sqrt{3\left(3+\sin ^{2} \phi\right)}} \\
& k=\frac{3 c}{\sqrt{9+12 \tan ^{2} \phi}}=\frac{3 c \cos \phi}{\sqrt{3\left(3+\sin ^{2} \phi\right)}}
\end{aligned}
$$

Eq. 19 and Eq. 20 demonstrate that it is the formula of DP3 criteria, derived by Drucker and Prager in 1951. It is just the MC match circle cirterion (DP) of plane strain under associated flow rule. In fact, it is the MC criterion under plane strain. At this time, the yield surface has transformed from hexagon to circle. Thus it is proved that the slope stability safety factor employing the criterion under plane strain is of high accuracy. Eq. 17 and Eq. 18 are formulas of MC match circle criterion (DP5) under plane strain applying nonassociated flow rule.

The MC match circle yield criteria under the two conditions applying plane strain mentioned above are of high accuracy. Attentions should be paid to that associated flow rule programs should be adopted under associated flow rule, and nonassociated flow rule programs conversely.

\section{SELECT OF DILATANCY ANGLE UNDER NONASSOCIATED AND ASSOCIATED FLOW RULE}

Plastic volumetric deformation occurs when particles in geomaterials dialocate due to shear, and the property is called dilatancy [12]. Dilatancy is a very important property of geomaterials. The select problem on dilatancy angle applying different flow rules under plane strain is discussed in the paper.

In discussing constitutive relations of materials, nonassociated flow rule is often employed for geomaterials and associated flow rule is often employed for metals. Generalized plastic theory [13-15] showed that associated flow rule is a special condition of nonassociated flow rule, and it suits materials whose plastic potential surface and yield surface are same, such as metals. Whatever in slip line field plastic theory, associated flow rule is often employed for both geomaterials and metals.

To fully understand the difference between associated and nonassociated flow rule in slip line field theory, the meanings of plastic potential surface, yield surface and failure surface should be made clear [8]. The normal direction of plastic potential surface demonstrates flow direction of materials, that is velocity vector direction, as its volumetric deformation is zero. For metals, the normal direction is sure to be the flow direction of shear deformation, that is direction of shear strain, or shear stress $q$, so the plastic potential surface is parallel to p-axial (Fig. 5). Yield surface is a surface where material stresses reaches yield state, and the surface is also parallel to p-axial for metals. So yield surface and plastic potential surface overlap, and associated flow rule can be adopted. For geomaterials, yield surface is an inclined plane, $\bar{\phi}$ from p-axial $\left(\bar{\phi}=3 \sin \phi /\left(\sqrt{3} \cos \theta_{\sigma}-\sin \theta_{\sigma} \sin \phi\right)\right)($ Fig. 5). So the yield surface of metals is different from that of geomaterials. For geomaterials, plastic potential surface is still parallel to p-axial, same as that of metals, that is, under the hypothesis that geomaterials are ideal elastic plastic materials with no volumetric deformation. At this time, the flow direction is still along $q$ direction. Influenced by traditional plastic mechanics, associated flow rule is often supposed to

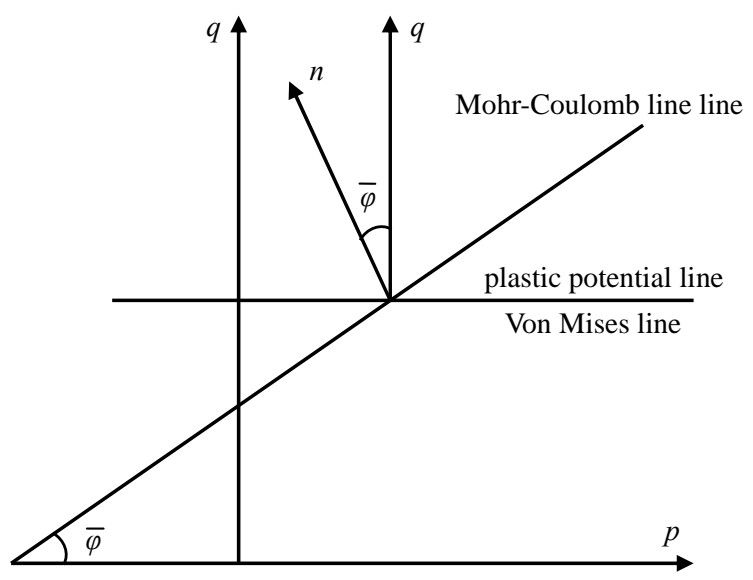

Fig. (5). Plastic potential and Mohr-Coulomb yield surfaces. 
be true for geomaterials. As plastic potential surface is supposed same as yield plane, and supposed to be an inclined line, $\bar{\phi}$ from $p$-axial, so corresponding flow direction is $\bar{\phi}$ from $q$-axial. Failur surface (stress slip line) is different from yield surface, and it expresses the true failure surface, that is stress slip line. For metals, it is an oblique line, $45^{\circ}$ from $\sigma$ axial (Fig. 6), and its direction is along q-direction. The direction of failure surface is same as the normal direction of yield surface and plastic potential surface. So it can be seen that velocity vector direction is sure to overlap with failure surface. For geomaterials, failure is an oblique line with $45^{\circ}+\phi / 2$ from $\sigma$ axial, and $\phi / 2$ from $q$ axial. It can be seen that velocity vector direction must be $\phi / 2$ from failure surface for geomaterials. Velocity vector direction is $\phi$ from failure surface according to slip line field theory under associated flow rule (Fig. 7).

\section{LAFE SOLUTION TO BEARING CAPACITY OF FOUNDATION}

The accuracy and applicability of LAFE, employed in calculation of bearing capacity of foundations should be rigorously put to the proof. The present work used LAFE to solve the classical Prandtl foundation bearing capacity problem which has accurate theoretical solutions for comparison. On one hand, by comparison with accurate theorectical solution, the accuracy and reliability of LAFE are proved, and on the other, the principles on determination of dilatancy angles and yield criterion advanced by the paper is perfectly reasonable and correct.

\section{Case 1}

In 1920, the accurate solution of the bearing capacity of semi-infinite rigid plastic foundation bearing homogeneous rigid vertical strip load, which assumes a weightless material, was advanced by Prandtl, according to plastic theory under associated flow rule. The formula is

$q_{u}=c N_{c}$

where $c$ is cohesion, and $N_{c}$ bearing capacity factor, with the expression of

$$
N_{c}=\cot \phi\left[\exp (\pi \tan \phi) \tan ^{2}\left(45^{\circ}+\frac{\phi}{2}\right)-1\right]
$$

The failure mechanism is composed of Rankine active area (I), radial shear area (II) and Rankine passive area (III) (Fig. 8).

Finite elements incremental loading was employed in calculation of the ultimate bearing capacity of the foundation with the mesh shown in Fig. (9). In calculation, adopting different criteria will bring different results. The yield criterion employed in ANSYS is external corner point circumscribed circle yield criterion(DP1). So equivalent transforms are of great necessity when different criteria are used. The transforming approach is equalling the values of $\alpha$ and $k$ of

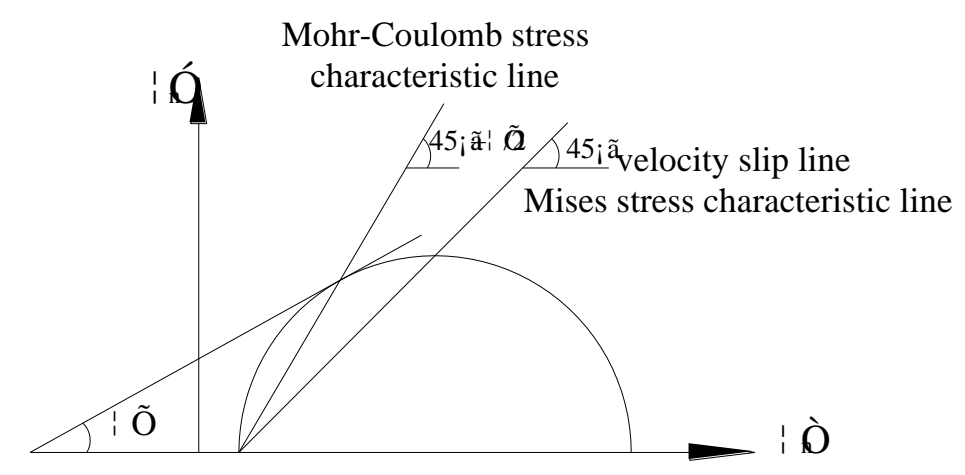

Fig. (6). Velocity slip line and stress characteristic line

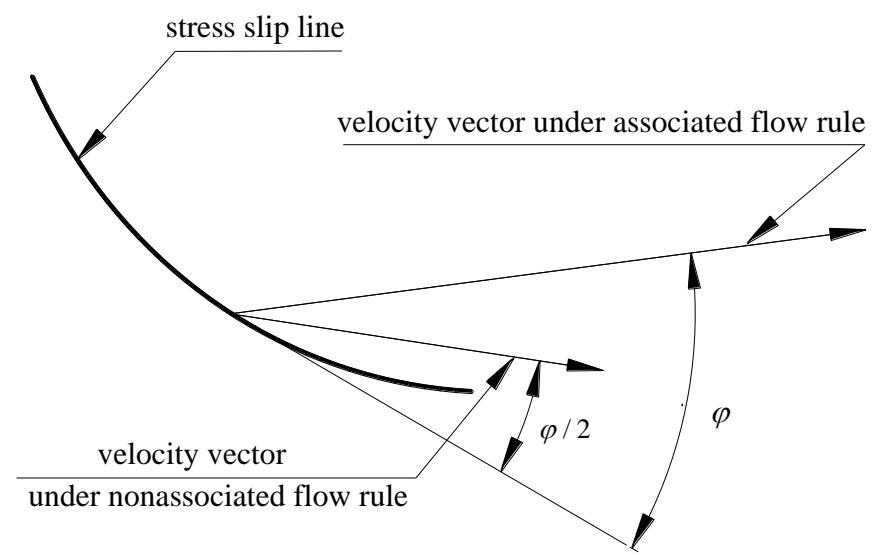

Fig. (7). Directions of velocity vector for associated and nonassociated flow rules 
different criteria (Table 1). For example, when MC match circle(DP5) employed, corresponding $\alpha$ and $k$ values for DP5 is obtained. Let the values of $\alpha$ and $k$ for DP1 equal to the values obtained. Then corresponding strength index values of $c$ and $\varphi$ for DP1 can be obtained by the formulae for DP1 listed in Table 1. Thus the DP5 criterion is realized by inputing the corresponding indexes for DP1 transformed through the above-mentioned approach.

\section{Results and Discussions}

According to the aforementioned approach, different yield criteria should be employed under different flow rules. Here MC inscribed circle yield criterion(DP3) is employed for associated flow rule and MC match circle(DP5) for nonassociated flow rule. The dilatancy angle of 0 and $\varphi / 2$ are selected respectively for comparison under the latter condition. The ultimate bearing capacity results under different conditions are listed in Table 2. Fig. (10a) demonstrates the plastic zone of limit state under associated flow rule $(\psi=\phi)$ with the strength parameters of $c=10 \mathrm{kPa}$ and $\phi=15^{\circ}$. Fig. $(\mathbf{1 0 b}, \mathbf{c})$ demonstrate the plastic zones of $\psi=0$ and $\psi=\phi / 2$ respectively under nonassociated flow rule with $c=10 \mathrm{kPa}$ and $\phi=15^{\circ}$. Corresponding displacement vector diagrams under the above three conditions are listed in Fig. (11a-c) respectively. The detailed failure mechanism under three conditions were carefully measured by hand from Fig. (10). Here the plastic area diagram were drawn by the actual size. The following parameters describing the failure mechanism (seen in Fig. 8) were surveyed in detail, which are included angle $\alpha$ between active Rankine area failure surface and horizontal plane, included angle $\beta$ between passive Rankine area failure surface and horizontal plane, plastic area width $L$ along horizontal direction above Rankine passive area (III) (Fig. 8), and plastic area depth $h$ along vertical direction and the depth $h_{0}$ of active Rankine area. Parameters of Prandtl solutions listed in Table $\mathbf{3}$ are strictly derived from analytical solutions.

Seen from Table 2, slip line mechanism parameters under associated flow rule and nonassociated flow rule $(\psi=\phi / 2)$ are perfectly identical to that by Prandtl theoretical solution, which indicates that the obtained plastic area diagrams under the two conditions are same as that by Prandtl solution. What's more, the ultimate bearing capacity obtained under the two conditions are also identical to that by Prandtl, with the errors less than $3 \%[16,17]$. On one hand, the correct-

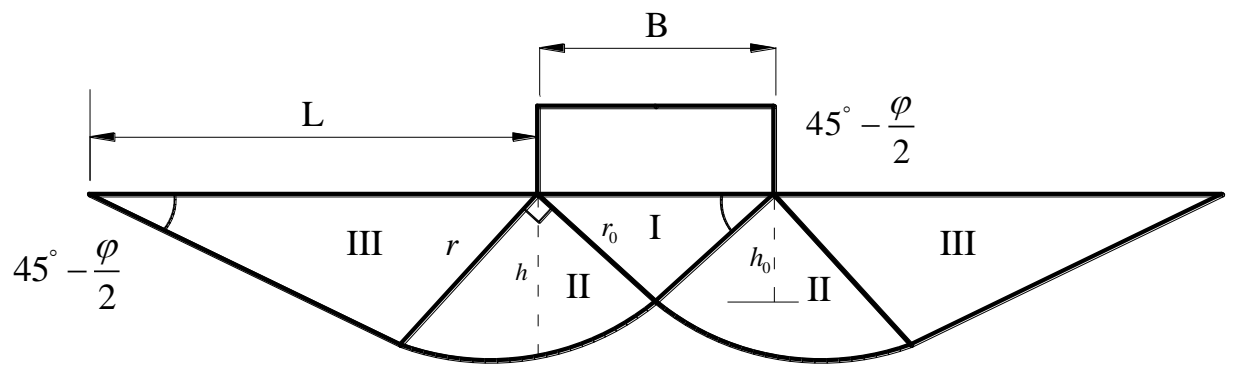

Fig. (8). Geometric pattern for Prandtl's solution.

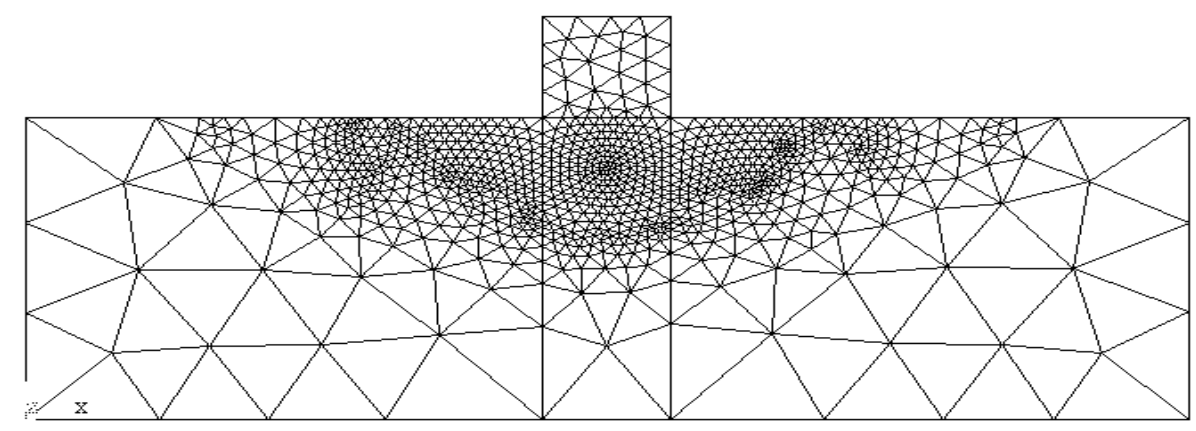

Fig. (9). Finite elements mesh.

Table 2. Comparative Ultimate Bearing Capacity Results of Prandtl Solution with those Obtained Under Associated and Nonassociated Flow Rule

\begin{tabular}{|c|c|c|c|c|c|c|}
\hline $\begin{array}{c}\text { strength } \\
\text { indexes }\end{array}$ & $\begin{array}{c}\text { Prandtl solu- } \\
\text { tion }\end{array}$ & \multicolumn{2}{|c|}{$\begin{array}{c}\text { DP3 }(\boldsymbol{\psi}=\boldsymbol{\varphi}) \\
\text { (associated flow rule) }\end{array}$} & $\begin{array}{c}\text { DP5 ( } \boldsymbol{\psi}=\mathbf{0}) \\
\text { (nonassociated flow rule) }\end{array}$ & $\begin{array}{c}\text { DP5 }(\boldsymbol{\psi}=\boldsymbol{\varphi} / \mathbf{2}) \\
\text { (nonassociated flow rule) }\end{array}$ \\
\hline \hline$\Phi=0^{\circ}$ & 51.4 & 52.2 & $1.6 \%$ & 52.2 & $1.6 \%$ & 52.2 \\
\hline$\varphi=15^{\circ}$ & 109.8 & 111.9 & $1.9 \%$ & 110 & $0.3 \%$ & 112.4 \\
\hline$\varphi=25^{\circ}$ & 207.2 & 212.1 & $2.4 \%$ & 201.7 & $-2.7 \%$ & $2.3 \%$ \\
\hline
\end{tabular}


ness of Prandtl solution is proved right once more, and on the other, the applicability and accuracy of finite elements incremental loading to calculate foundation bearing capacity. Nevertheless the only difference is the direction of velocity vector between the two conditions [18]. The velocity vector direction can not only be seen from dilatancy angle, but also be printed in detail from computation results. Similar conclusion is also available in literature [19].

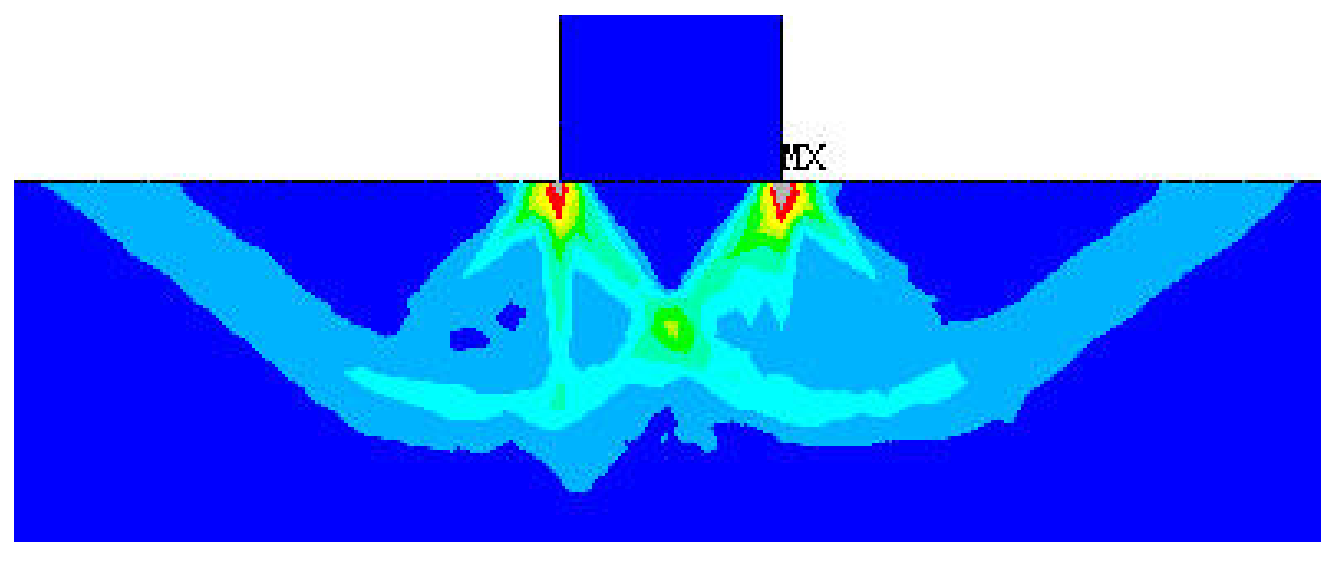

(a) $\psi=\phi$ (associated flow rule)

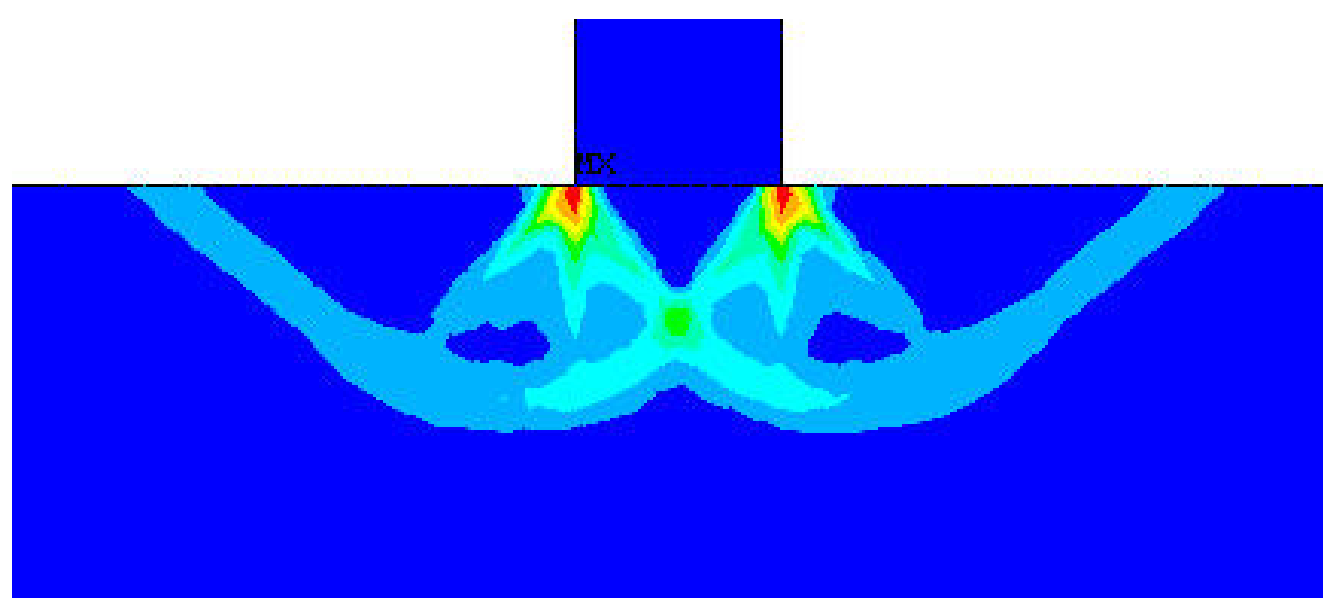

(b) $\psi=\phi / 2$ (nonassociated flow rule)

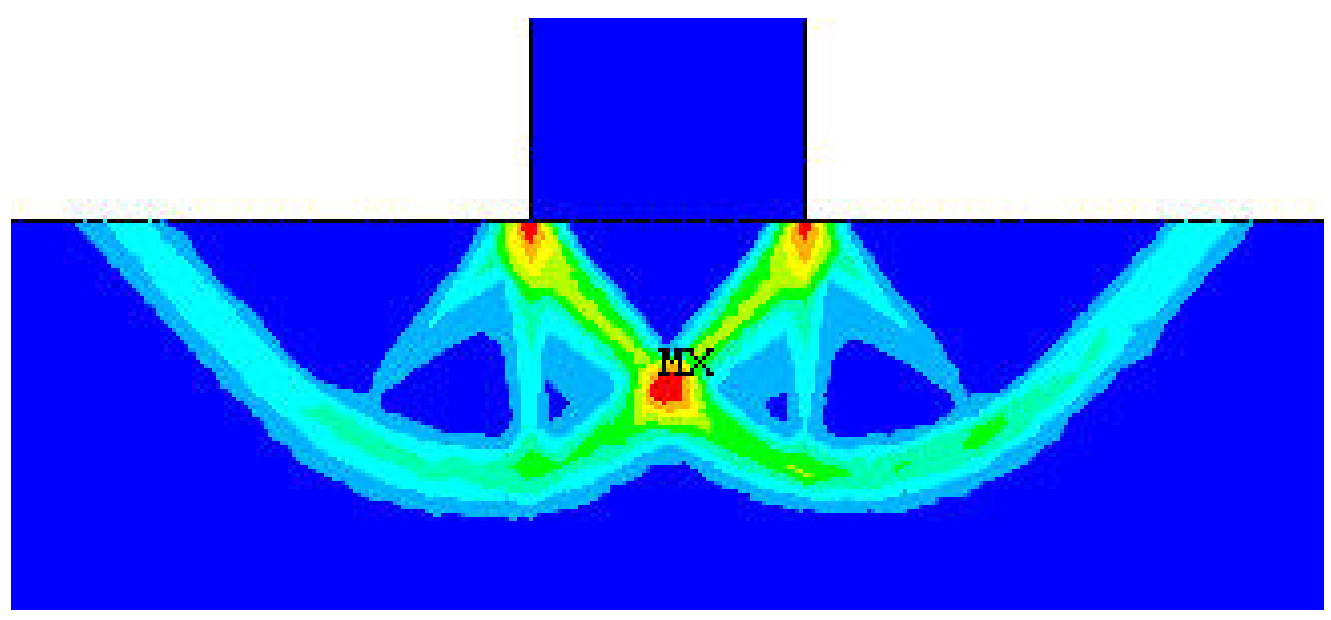

(c) $\psi=0$ (nonassociated flow rule)

Fig. (10). Failure surface under limit state. 
Table 3. Comparative Results Between Prandtl Solution and Finite Elements Results Under Different Flow Rules and Different Dilatancy Angles

\begin{tabular}{|c|c|c|c|c|}
\hline Comparing items & Prandtl Solution & $\operatorname{DP3}(\psi=\phi)$ & $\operatorname{DP5}(\psi=0)$ & $\operatorname{DP5}(\psi=\phi / 2)$ \\
\hline $\begin{array}{l}\text { Inclined angle between slip plane of passive Rankine area and horizon- } \\
\text { tal plane }\end{array}$ & $37.5^{\circ}$ & $37^{\circ}$ & $46^{\circ}$ & $37.5^{\circ}$ \\
\hline Horizontal range of plastic area $L$ & $1.99 \mathrm{~B}$ & $2 \mathrm{~B}$ & $1.61 \mathrm{~B}$ & $2 \mathrm{~B}$ \\
\hline
\end{tabular}

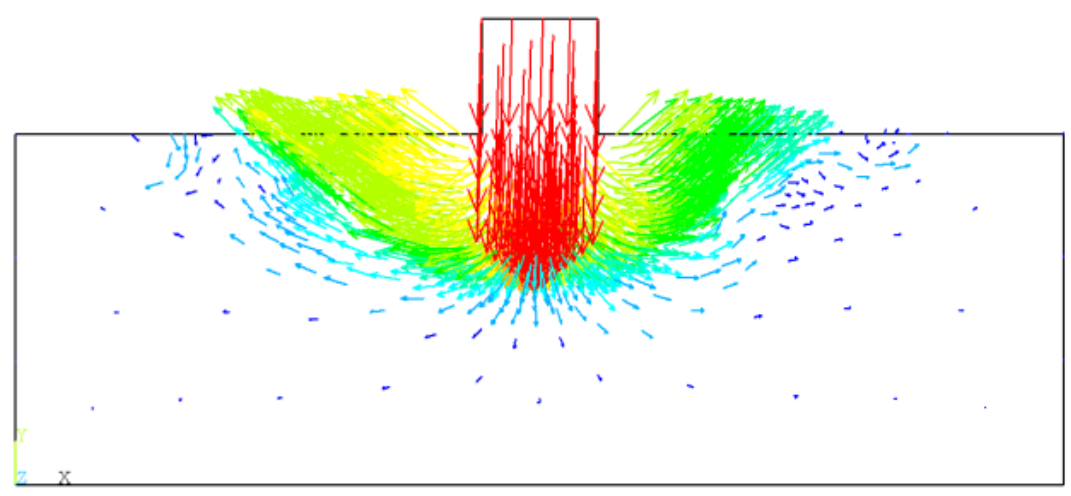

(a) $\psi=\phi$ (associated flow rule)

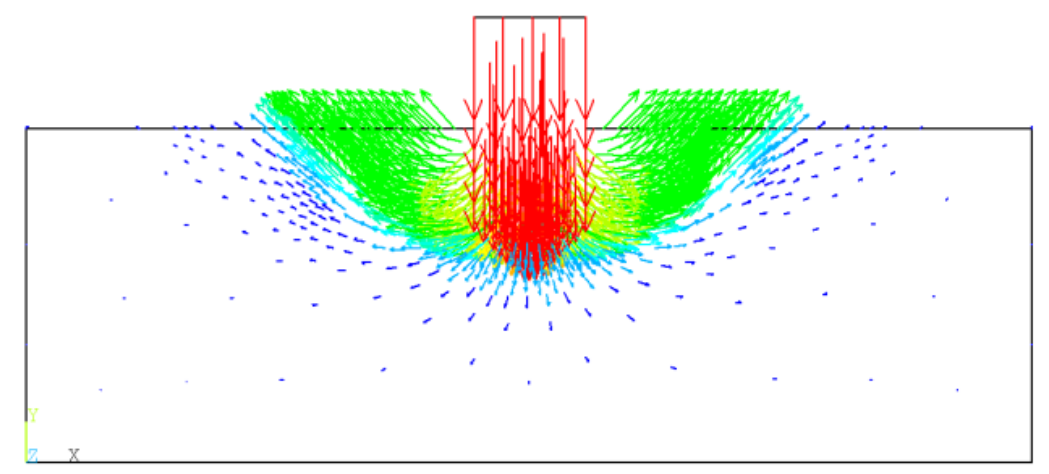

(b) $\psi=\phi / 2$ (nonassociated flow rule)

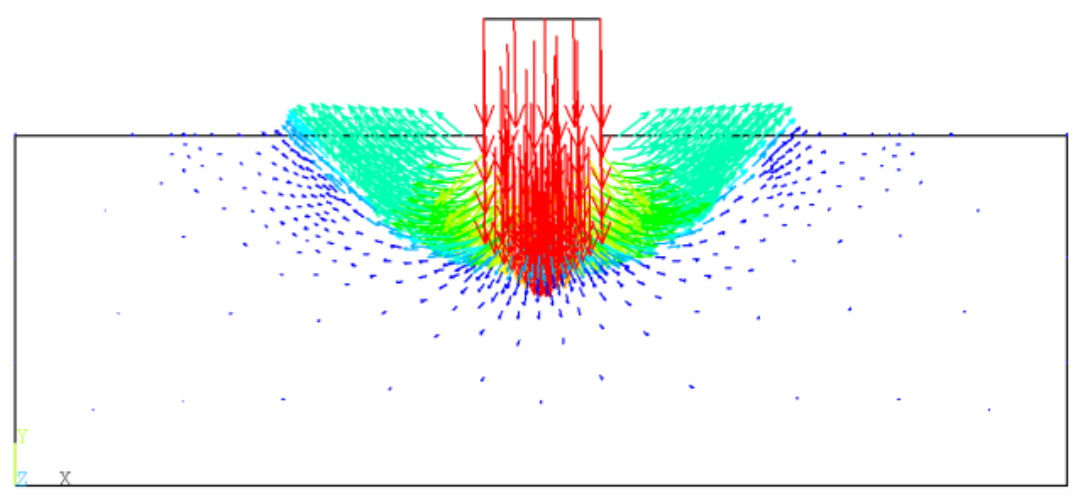

(c) $\psi=0$ (nonassociated flow rule)

Fig. (11). Displacement vector under limit state. 
Seen from Tables $\mathbf{2}, \mathbf{3}$ and Fig. (8), the close solution of ultimate bearing capacity is also obtained under nonassociated flow rule $(\psi=0)$, but with a large error of slip line field compared with the theoretical solution.

\section{CONCLUSIONS}

Finite elements incremental loading is put forward to solve bearing capacity problem of foundation, and this approach is successfully implemented. Former researches have discussed the problem, but with a large error, which made it hard to be used in engineeing practice. The present work makes a high accuracy for the first time.

The paper proved again that same ultimate bearing capacity and slip line are obtained in slip line field theory under associated and nonassociated flow rule, with the only difference of velocity vector direction. Meanwhile, the dilatancy angle should be $\varphi / 2$ when nonassociated flow rule is employed under plane strain, and corresponding volumetric strain is zero. Thus the correctness of the theoretical solution in literature [19] is proved, and LAFE method is also proved a very promising approach to solve bearing capacity problems of foundations.

Rigorous theoretical basis are available for finite elements incremental loading to solve the bearing capacity problems of foundations, and the approach is simple to use. In the numerical simulation process, not only the ultimate bearing capacity and load-displacement curve are obtained, but also the failure mechanism proved same as the one by traditional limit analysis approach is achieved. Attention should be paid to the select of the yield criterion in the process. Only the yield criterion matched with practical engineering problems can generate a precise result. Under plane strain the results by Mohr-Coulomb inscribed circle yield criterion (DP3) for associated flow rule, and Mohr-Coulomb match yield criterion (DP5) for nonassociated flow rule are close to the accurate theoretical solution by Prandtl. The achievements can be applied in practical geotechnical engineering purposes.

\section{ACKNOWLEDGEMENTS}

This work was financially supported by Open Fund of Engineering Research Center of Catastrophic Prophylaxis and Treatment of Road \& Traffic Safety(Changsha University of Science \& Technology), Ministry of Education (kfj080302).

\section{REFERENCES}

[1] W. F. Chen. Limit analysis and soil plasticity. Amsterdam: Elsevier, 1975
[2] Y. R. Zheng, S. Y. Zhao, A. H. Li, et al. "FEM limit analysis and its application in slope engineering. Beijing: China Communications Press, 2011(in Chinese)

[3] D. C. Drucker, W. Prager, "Soil mechanics and plastic analysis on limit design", Quarterly Applied Mathematics, vol. 10, pp. 157-65, 1952.

[4] Y. R. Zheng, S. Y. Zhao, "Limit state finite element method for geotechnical engineering analysis and its applications", Chinese Civil Engineering Journal, vol. 38, no. 1, pp. 91-99, 2005 (in Chinese)

[5] Y. R. Zheng, S. Y. Zhao, W. X. Kong, et al. Geotechnical engineering limit analysis using finite element method[J]. Rock and Soil Mechanics, vol. 26, no. 1, pp. 163-168, 2005 (in Chinese)

[6] C. J. Deng, W. X. Kong and Y. R. Zheng. "Analysis of ultimate bearing capacity of foundations by elastoplastic FEM through step loading", Rock and Soil Mechanics, vol. 26, no. 3, pp. 500-504, 2005 (in Chinese)

[7] W. X. Kong, C. J. Deng, Y. Q. Rui, et al. "FEM analysis of associated and nonassociated flow rules in theory of slip line field", Journal of Northeastern University (Natural Science), vol. 28, no. 3, pp. 430-453, 2007. (in Chinese)

[8] W. X. Kong, Weakening Effect of Water on Rock and Soil Massed and Bearing Capacity Stability Problems of Foundations in the Three Georges Reservoir Area[D]. Chongqing: Logistical University, 2005 (in Chinese)

[9] W. X. Kong, Y. Q. Rui and B. D. Dong. "Determination of dilatancy angle for geomaterials under nonassociated flow rule", Rock and soil mechanics, vol. 30, no. 11, pp. 3278-3282, 2009 (in Chinese)

[10] Y. R. Zheng, S. Y. ZHAO, "Application of strength reduction FEM in soil and rock slope", Chinese Journal of Rock Mechanics and Engineeringx, vol. 23, no. 19, pp. 3381-3388, 2009 (in Chinese) (in Chinese)

[11] G. C. XU, Y. R. Zheng, "Research on yield criterion in rock engineering", Chinese Journal of Geotechnical Engineering, vol. 1990, no. 2, pp. 93-99, 1990 (in Chinese)

[12] C. J. Deng, Y. R. Zheng and K. Wang, "Some discussion of the dilatancy of geotechnical materials, Beijing", Chinese Journal of Geotechnical Engineering, vol. 31, no. 7, pp. 1110-1114, 2009 (in Chinese)

[13] Y. R. Zheng, Z. J. Shen and X. N. Gong, "Geotechnical plastic mechanics principles-Generalized plastic mechanics. Beijing", China Building Industry Press, 2002 (in Chinese)

[14] Y. R. Zheng, L. Kong. Geotechnical plastic mechanics. Beijing: China Building Industry Press, 2010 (in Chinese)

[15] Y. R. Zheng, L. Kong, "Generalized plastic mechanics and its application", Engineering Science, vol. 7, no. 11, pp. 21-36, 2005 (in Chinese)

[16] L. Prandtl. "Über die Härter plastischer Körper Nachrichten von der Geselschaft der Wissenschaften zu Göttingen“, Mathematical Physics Klasse, vol. 12, pp. 74-85, 1920

[17] X. Y. Zhang, "Plastic mechanics slip line theorem for Prandtl and Terzaghi ultimate bearing capacity of foundations", Journal of Tianjin University, vol. 20, no. 2, pp. 22-29, 1987 (in Chinese)

[18] M. Hjiaj, A. V. Lyamin and S. W. Sloan, "Numerical limit analysis solutions for the bearing capacity factor $N_{\gamma}$, International Journal of Solids and Structures, vol. 42, no. pp. 1681-1704. 2005.

[19] Y. R. Zheng, J. L. Wang and X. K. Zhu, "Discussion on velocity solution of slip line theory for geotechnical materials". Journal of Hydraulic Engineering, vol. 2001, no. 6, pp. 1-7, 2001 (in Chinese) 